\title{
Faces of phase
}

\author{
Prem Soman, MD, PhD, FRCP (UK), FACC, FASNC ${ }^{a}$ \\ a Division of Cardiology and the Heart and Vascular Institute, University of Pittsburgh Medical \\ Center, Pittsburgh, PA
}

Received Jul 24, 2018; accepted Jul 24, 2018

doi:10.1007/s12350-018-1390-9

\section{See related article, pp. 575-588}

In this issue of the Journal, Van Tosh and colleagues ${ }^{1}$ report on a novel application of ventricular dyssynchrony measured by phase analysis of regional myocardial thickening curves. They apply this technique to the detection of obstructive coronary stenosis in individual vessels and the diagnosis of multi-vessel disease in patients. The concept is intriguing and the results, somewhat surprising. Nevertheless, with this report the investigators reveal yet another "face", namely its diagnostic value for obstructive $\mathrm{CAD}$, of phase analysis which has hitherto been shown to predict response to cardiac resynchronization therapy (CRT), ${ }^{2,3}$ ventricular arrhythmia in patients with LV systolic dysfunction, ${ }^{4}$ and prognosis in patients with CAD or heart failure. ${ }^{5}$

The application of phase analysis to radionuclide myocardial perfusion images leverages the phenomenon of partial volume effect due to which there is a relatively linear relationship between myocardial thickening and regional radiotracer count intensity during a cardiac cycle. ${ }^{6}$ Thus, a time activity curve of a myocardial segment is essentially its thickening curve. Fourier transformation produces a continuous thickening curve of high temporal fidelity and allows the identification of the phase of the onset of mechanical contraction (OMC), phase of peak contraction (peak phase), and phase amplitude. A phase histogram can be constructed using either the phase of OMC or peak phase from which the established measures of systolic mechanical

Reprint requests: Prem Soman, MD, PhD, FRCP (UK), FACC, FASNC, Division of Cardiology and the Heart and Vascular Institute, University of Pittsburgh Medical Center, A-429 Scaife Hall, 200 Lothrop Street, Pittsburgh, PA 15213; premsoman@usa.net J Nucl Cardiol 2020;27:589-91.

$1071-3581 / \$ 34.00$

Copyright (C) 2018 American Society of Nuclear Cardiology. dyssynchrony, namely phase histogram bandwidth and phase standard deviation are calculated. ${ }^{7}$ Prior studies have established good precision. ${ }^{8}$ A similar approach has recently been applied to the measurement of diastolic (dys)synchrony using the onset of mechanical relaxation (OMR). ${ }^{9}$

While much of the literature on radionuclide methods of dyssynchrony assessment relates to singlephoton emission computed tomography (SPECT) and radionuclide ventriculography, the principle can be applied equally to positron-emission tomography (PET). ${ }^{10}$ Van Tosh and colleagues applied phase analysis to PET perfusion scans from 105 patients with known (including prior $\mathrm{CABG}$ ) or suspected $\mathrm{CAD}$ who had previously undergone $\mathrm{Rb}-82$ myocardial perfusion imaging with regadenoson stress and coronary angiography. Angiograms were analyzed quantitatively at a core laboratory, which is a strength of this study. However, invasive functional measurements such as fractional flow reserve or instant wave-free ratio were not performed. Segmental myocardial blood flow (MBF) and myocardial flow reserve (MFR) were calculated from dynamic PET data. Ventricular systolic and diastolic synchrony were measured using the OMC and OMR, respectively. The investigators then derived a complex measure of "asynchrony" by scoring each coronary territory on a 5-point scale for rest systole, rest diastole, stress systole, and stress diastole taking into consideration the width and timing of the histograms and the uniformity of the phase polar maps. A detailed description of this methodology is lacking in the paper. A major advantage of the radionuclide approach to dyssynchrony assessment is its largely automated nature and thus, high precision. By employing a complicated and somewhat subjective score rather than using a standard computer derived metric such as the histogram bandwidth or phase standard deviation, the investigators lose some of this advantage. The stated objective of this study was to compare the accuracy of flow measurements to other parameters including the asynchrony score for the detection of individual coronary stenosis 
$\geq 70 \%$ and the presence of multi-vessel disease ( $\geq 70 \%$ in $\geq 2$ coronary territories).

Among the 105 patients studied, quantitative coronary angiography revealed 47 patients (44\%), 65 vessels with $\geq 70 \%$ stenosis and 15 patients with multi-vessel disease. Thus, the prevalence of disease was acceptable for a diagnostic accuracy study. Quite surprisingly, the asynchrony score outperformed quantitative flow measurements and relative perfusion imaging scores for identifying individual coronary stenosis. The area under the curve (AUC) for the receiver operating characteristics (ROC) curve was $81 \pm 3 \%$ and $72 \pm 4 \%$ for the asynchrony score and MFR $<1.88$, respectively (data for the other covariates are presented in Table 4). For patient level data, the asynchrony score was superior (AUC $84 \% \pm 6 \%$ ) to stress MFR $<1.76$ (AUC $73 \% \pm 7 \%$ ), change in EF from rest to stress $<4 \%$ (AUC $66 \mathrm{~T} \pm 8 \%$ ), transient ischemic dilatation $(56 \% \pm 7 \%)$ and perfusion scores for the identification of multi-vessel disease, but similar to stress $\mathrm{MBF}<$ 0.94 and stress $\mathrm{EF}<24 \%$ (data presented in Table 3). These specific cut-offs were chosen based on the value that optimized the Youden Index for the AUC of the ROC curve.

Is there a physiologically tenable explanation for the surprisingly high and superior accuracy of the asynchrony score for the detection of individual coronary stenosis and multi-vessel disease? While the authors make no attempt at a mechanistic explanation for their findings, a consideration of this question requires some speculation about the pathobiology of regional LV dyssynchrony due to CAD. One possibility is that regional $\mathrm{LV}$ dysfunction induced by myocardial ischemia results in dyssynchrony. In this regard, PET has the advantage of being able to acquire LV function data at peak vasodilator stress in contrast to SPECT where image acquisition is delayed by several minutes after peak stress by which time ischemic regional dysfunction may have resolved. Accordingly, other PET but not SPECT studies have shown an association between ischemia and LV dyssynchrony. ${ }^{11,12}$ However, unlike exercise, vasodilators only infrequently produce myocardial ischemia and regional dysfunction and do so either by coronary steal or by reducing diastolic blood pressure and coronary perfusion. ${ }^{13}$ Thus, this mechanism is unlikely to be the predominant explanation for the link between LV dyssynchrony and obstructive CAD seen in this and other studies.

The second possibility is that the presence of LV dyssynchrony implies extensive CAD and LV systolic dysfunction mediated by hibernation and infarction. The finding that severe LV systolic dysfunction $(\mathrm{EF}<24 \%)$ had a similar AUC as the asynchrony score $(80 \pm 7 \%$ vs. $84 \pm 5 \%, P=\mathrm{ns}$ ) for identifying patients with multivessel disease supports this postulate. However, it does not explain the accuracy of the asynchrony score for identifying individual coronary stenosis or the poor sensitivity of resting perfusion scores for identifying multi-vessel disease.

Finally, the results may be partly related to the imperfect relationship between quantitative measures of myocardial blood flow and obstructive coronary stenosis such that there is only a modest diagnostic accuracy of stress MBF and MFR for obstructive CAD defined by an anatomical standard.

The important question is whether the asynchrony score proposed by the authors can be used as a pragmatic indicator of multi-vessel CAD. Dyssynchrony measures are obtained from a standard myocardial perfusion scan with no additional requirements and thus, can be easily applies to patients referred for stress testing. However, several aspects of this report from van Tosh may preclude generalization of their data. First, the study population was highly selected in that patients had to have undergone both PET and coronary angiography and therefore were skewed towards higher risk. It remains to be seen if this data can be replicated in allcomers to the PET lab. Second, the asynchrony score described by the authors lacks sufficient detail for replication, is complex, and somewhat subjective. If automated measures of dyssynchrony can be substituted for the asynchrony score, the approach may be more widely applicable. Finally, the cut-off values for the covariates used (asynchrony score cut-offs of $>26$, $>28$, and $>6$, and MFR cut-offs of $<1.76,<1.71$ and $<1.88$ were used to diagnose two-vessel, three-vessel, and individual vessel CAD, respectively) were generated from the study population itself and may not be applicable to other populations.

The detection of multi-vessel disease is a challenge with relative perfusion imaging and difficult even when quantitative blood flow measurements are available. Despite many limitations, this study from Van Tosh and colleagues offers us a glimpse of a potentially useful application of PET dyssynchrony assessment to the challenging clinical problem of identifying patients with multi-vessel CAD. However, before clinical application can be recommended, the data must be replicated in patients with a broader range of CAD risk and by using a simpler measure of LV dyssynchrony.

\section{Disclosure}

Prem Soman has nothing to disclose. 


\section{References}

1. Van Tosh A, Votaw JR, David Cooke C, et al. Relationship of ${ }^{82} \mathrm{Rb}$ PET territorial myocardial asynchrony to arterial stenosis. J Nucl Cardiol 2018. https://doi.org/10.1007/s12350-018-1350-4.

2. Friehling M, Chen J, Saba S, et al. A prospective pilot study to evaluate the relationship between acute change in left ventricular synchrony after cardiac resynchronization therapy and patient outcome using a single-injection gated SPECT protocol. Circ Cardiovasc Imaging 2011;4:532-9.

3. Boogers MM, Van Kriekinge SD, Henneman MM, et al. Quantitative gated SPECT-derived phase analysis on gated myocardial perfusion SPECT detects left ventricular dyssynchrony and predicts response to cardiac resynchronization therapy. J Nucl Med 2009;50:718-25.

4. Malhotra S, Pasupula DK, Sharma RK, Saba S, Soman P. Relationship between left ventricular dyssynchrony and scar burden in the genesis of ventricular tachyarrhythmia. J Nucl Cardiol 2018;25:555-69.

5. Fudim M, Fathallah M, Shaw LK, et al. The prognostic value of diastolic and systolic mechanical left ventricular dyssynchrony among patients with coronary heart disease. JACC 2018. https://d oi.org/10.1016/j.jcmg.2018.05.018.

6. Galt JR, Garcia EV, Robbins WL. Effects of myocardial wall thickenss on SPECT quantification. IEEE Trans Med Imaging 1990;9:144-50.

7. Chen J, Garcia EV, Bax JJ, Iskandrian AE, Borges-Neto S, Soman P. SPECT myocardial perfusion imaging for the assessment of left ventricular mechanical dyssynchrony. J Nucl Cardiol 2011;18:685-94.

8. Lin $\mathrm{X}, \mathrm{Xu} \mathrm{H}$, Zhao $\mathrm{X}$, et al. Repeatability of left ventricular dyssynchrony and function parameters in serial gated myocardial perfusion SPECT studies. J Nucl Cardiol 2010;17:811-6.

9. Boogers MJ, Chen J, Veltman CE, et al. Left ventricular diastolic dyssynchrony assessed with phase analysis of gated myocardial perfusion SPECT: a comparison with tissue Doppler imaging. Eur J Nucl Med Mol Imaging 2011;38:2031-9.

10. Cooke CD, Esteves FP, Chen J, Garcia EV. Left ventricular mechanical synchrony from stress and rest $82 \mathrm{Rb}$ PET myocardial perfusion ECG-gated studies: differentiating normal from LBBB patients. J Nucl Cardiol 2011;18:1076-85.

11. Aljaroudi W, Koneru J, Heo J, Iskandrian AE. Impact of ischemia on left ventricular dyssynchrony by phase analysis of gated single photon emission computed tomography myocardial perfusion imaging. J Nucl Cardiol 2011;18:36-42.

12. AlJaroudi W, Alraies MC, Menon V, Brunken RC, Cerqueira MD, Jaber WA. Predictors and incremental prognostic value of left ventricular mechanical dyssynchrony response during stress-gated positron emission tomography in patients with ischemic cardiomyopathy. J Nucl Cardiol 2012;19:958-69.

13. Soman P, Lahiri A, Senior R. Vasodilator stress induces infrequent wall thickening abnormalities compared to perfusion defects in mild-to-moderate coronary artery disease: implications for the choice of imaging modality with vasodilator stress. Echocardiography 2004;21:307-12. 